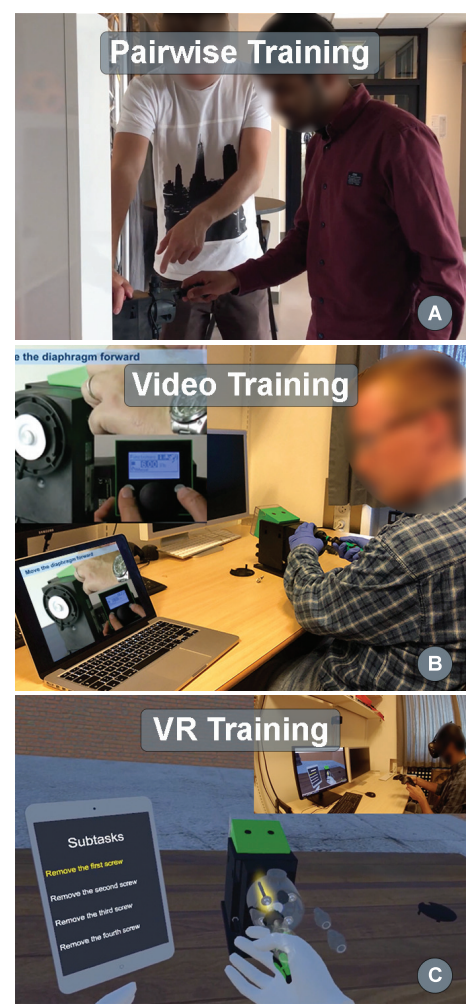

Figure 1: Based on a use case at Grundfos, we compared 3 methods for training novice technicians in a structured maintenance task: (A)

Pairwise Training, (B) Video Training, and (C) VR Training.

\title{
Design and Evaluation of a VR Training Simulation for Pump Maintenance
}

Frederik Winther $\ddagger$

Aarhus University

Denmark

Linoj Ravindran $\ddagger$

Aarhus University

Denmark

Kasper Paabøl Svendsen

Aarhus University

Denmark

Tiare Feuchtner

Aarhus University

Denmark

‡ Indicated authors contributed equally to this research.

Permission to make digital or hard copies of part or all of this work for personal or classroom use is granted without fee provided that copies are not made or distributed for profit or commercial advantage and that copies bear this notice and the full citation on the first page. Copyrights for third-party components of this work must be honored. For all other uses, contact the owner/author(s).

CHI '20 Extended Abstracts, April 25-30, 2020, Honolulu, HI, USA.

Copyright is held by the author/owner(s).

ACM ISBN 978-1-4503-6819-3/20/04.

http://dx.doi.org/10.1145/3334480.3375213

\begin{abstract}
Through technological advancements more and more companies consider virtual reality (VR) for training of their workforce, in particular for situations that occur rarely, are dangerous, expensive, or very difficult to recreate in the real world. This creates the need for understanding the potentials and limitations of VR training and establish best practices. In pursuit of this, we have developed a VR Training simulation for a use case at Grundfos, in which apprentices learn a sequential maintenance task. We evaluated this simulation in a user study with 36 participants, comparing it to two traditional forms of training (Pairwise Training and Video Training). This case study describes the developed virtual training scenario and discusses design considerations for such VR simulations. The results of our evaluation support that, while VR Training is effective in teaching the procedure for a maintenance task, traditional approaches with hands-on experience still lead to a significantly better outcome.
\end{abstract}

\section{Author Keywords}

Virtual reality; training; industrial manufacturing.

\section{CCS Concepts}

-Human-centered computing $\rightarrow$ User studies; Virtual reality; 


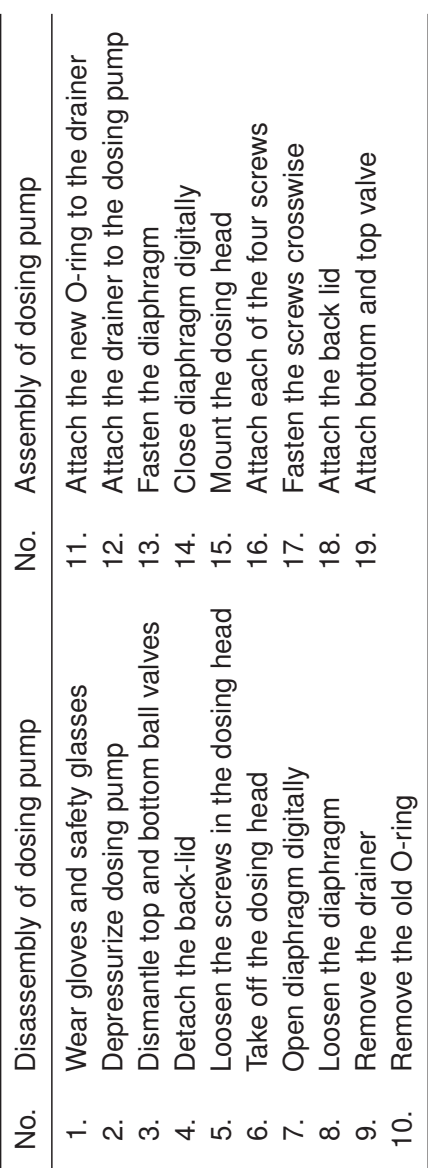

Table 1: Sequence of steps that need to be carried out in order to change the o-ring on the dosing pump.

\section{Method}

In industrial research, Virtual Reality (VR) holds the promise of potential low-cost solutions for design and prototyping of new products and simulation of processes for knowledge gain [1]. An obvious advantage of using VR as a training tool, is that it provides a safe environment for making mistakes and makes it possible to practice scenarios that might not occur very often or are difficult to simulate in the real world $^{1}$. Furthermore, in VR processes can be trained involving machinery and tools that are physically unavailable (e.g., since stopping the production line is not desirable), or because the setup might not be accessible or not even exist yet (e.g., training for space missions). Finally, VR (as well as augmented reality) offers the opportunity of supplying information in an engaging fashion that is not possible in the real world, e.g., highlighted objects and animations for guidance, instead of paper manuals.

However, there is a lack of long-term, large-scale evaluations that provide evidence for the effectiveness of VR simulations as a training tool. Arguably, not all forms of learning can be equally well supported by VR simulations (e.g., memorizing a sequence of clearly defined steps by practicing vs. exploring material properties and tools when sculpting a statue). Furthermore, we cannot assume that our lessons learned for the design of traditional computer interfaces will apply equally in this immersive 3-dimensional domain, so there is also a need for new design guidelines and best practices.

We explore a case at Grundfos, where apprentices are taught a sequential maintenance task for many different pump models. The company employs various different modes and materials to instruct their workers, such as pair-

${ }^{1}$ Walmart Black Friday training (accessed: Oct.14,2019): https://www.businessinsider.de walmart-using-virtual-reality-employee-training-2017-6 wise training and instructional videos. Training of this task traditionally poses the following challenge: either a dosing pump needs to be shut down and uninstalled from the system, or an additional dosing pump must be acquired and made explicitly available for training purposes. Here VR presents a valuable opportunity for training, without interfering with the system in operation or requiring an instructor to be available, while still providing near-realistic manipulation of the pump and its sub-components in 3D space. The challenge however, lies in evaluating how effective such VR simulations are in teaching sequential tasks, in what cases they may be beneficial or detrimental, and whether they are fit to replace or rather complement traditional forms of training. Thus, we explore the effectiveness of $V R$ Training for a maintenance task on a dosing pump, in comparison to traditional training methods (Video Training, Pairwise Training) in a user study (see training methods in Figure 1). Further, we present several design considerations for the creation of this VR training simulation.

\section{Training simulation for a maintenance task}

Description of the use case

Companies such as Grundfos mainly apply two different training methods to teach new employees how to handle the machinery: Pairwise Training and VR Training. In the first, a trainee is personally educated by an instructor. The latter is carried out in a dedicated room, where the trainee is equipped with VR gear and completes the training independently. In this particular case, each VR Training session takes about 20 minutes. The task is divided into a series of steps. In each of these narration and visual highlights inform the trainee with which object needs to be interacted and how. Trainees interact with the simulation by selecting and manipulating the highlighted object with their controllers. Apart from these two training techniques, instruc- 


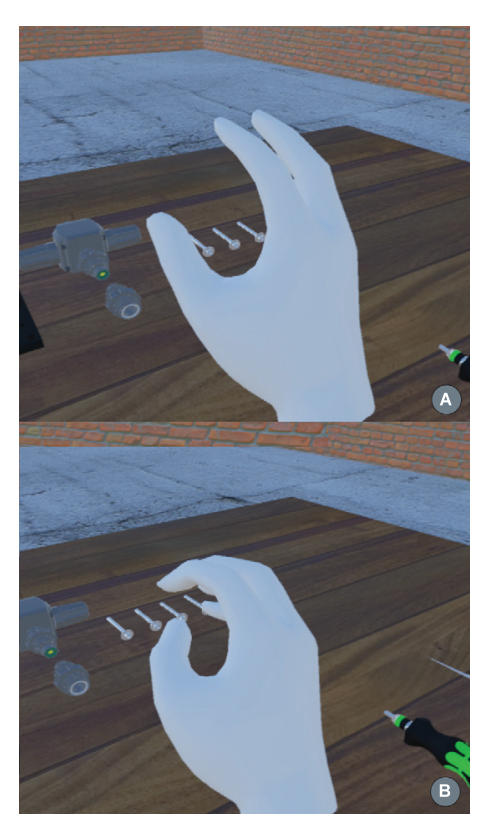

Figure 2: In the VE virtual hands can pick up interactive

components. (A) Relaxed hand with extended fingers, when the user is not grabbing. (B) Pulling the trigger on the controller causes the hand to grab. tional videos are often available as a further aid when practicing maintenance tasks.

Interestingly, many training experts at companies like Grundfos consider Pairwise Training and VR Training as equally effective training methods, without any supporting evidence. To gain such evidence, we developed a simulation of an exemplary maintenance task. This task involves changing the o-ring of a dosing pump, which requires several steps for disassembly and reassembly (see Table 1). The VR Training simulation was designed to closely follow the predefined steps for one specific pump, for which an instructional video was available for comparison.

\section{Technical Setup and Interaction Design}

The VR Training simulation was developed in Unity $3 \mathrm{D}^{2}$ and experienced through an HTC Vive ${ }^{3}$ head mounted display (HMD). Matching HTC Vive controllers served as input devices for interaction in the virtual environment (VE). The training simulation was run on a Windows PC with a GeForce GTX 1070 Graphics Card.

We followed an iterative design process involving a series of pilot studies. These served to test out different aspects of the prototype, such as how hints are presented and how selected objects are visualized in the VE.

In the resulting prototype the user interacts through two virtual hands (3D-hand-models that appear to be wearing white gloves). These hands follow the movements of the Vive Controllers, thereby reflecting the user's own hand movements. Further, when the user pulls the trigger on a controller, the fingers of the respective virtual hand curl inward, as if grabbing something (see Figure 2). This suggests to the user that they can simply reach out and grab objects in the VE to manipulate them. Such a hand selec-

\footnotetext{
2Unity 3D https://unity.com/

${ }^{3} \mathrm{HTC}$ Vive HMD https://www.vive.com/us
}

tion pattern generates expectations, e.g., that objects in the virtual world behave like in the real world [5, Chapter 28].

To contextualize the training, the VE resembles an industrial facility (see Figure 3, A). A special scene was designed, to permit users to get acquainted with VR and freely explore the VE prior to the actual training. They hereby playfully acquire the skills for manipulations that are needed in training. The virtual training scenario aims at simulating a real-world maintenance task to decrease contextual changing $[6,8]$, and includes a detailed 3D model of a dosing pump (supplied by Grundfos). Furthermore, there are two different tools (a torque screwdriver and a needle tool) with which users disassemble components, replace the o-ring, and then reassemble the dosing pump. A Guidance System, described in the following section, informs the user about the required steps for the task.

Apart from the dosing pump, all virtual content was obtained from Sketchup's 3D warehouse ${ }^{4}$ and modified, or specifically designed in Blender ${ }^{5}$.

\section{Multimodal Guidance System}

During the training a guidance system provides multimoda cues, including voice recordings, textual instructions, highlights and animations, and haptic feedback [7]. To improve knowledge transfer, two levels of difficulty were implemented [10]: Beginner and Intermediate. When training at beginner level the user is provided with as much assistance as possible. At intermediate level the amount of guidance is decreased by omitting animations and the highlights on objects, thereby requiring increased use of cognitive abilities.

Voice recordings and textual instructions: A female voice guides the user through the sequence of steps, providing instructions and important information regarding

\footnotetext{
${ }^{4}$ Sketchup's 3D warehouse https://3dwarehouse.sketchup.com/ ${ }^{5}$ Blender (version 2.79b) https://www.blender.org/ 

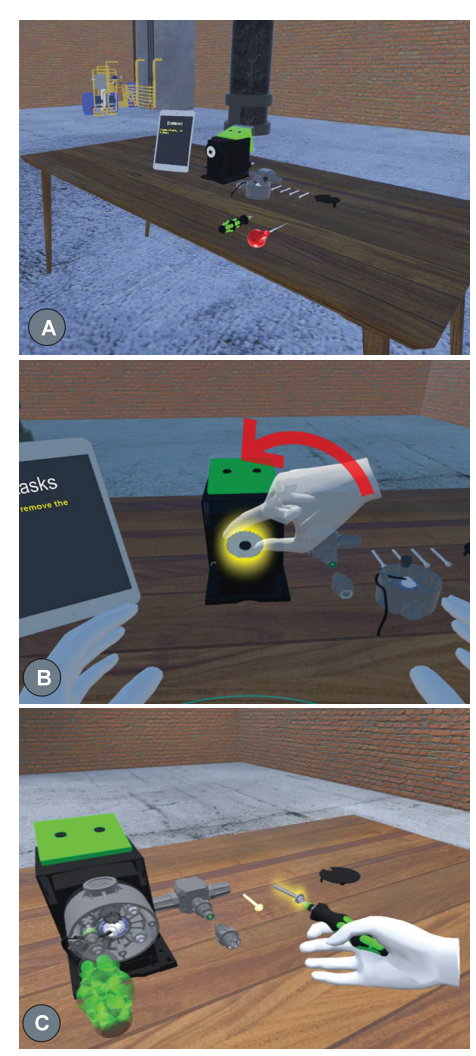

Figure 3: The VR simulation involves $(A)$ a dosing pump and tools on a workbench. (B) Animations and highlights provide step-by-step guidance (red arrow indicates animation of virtual hand). (C) Yellow target silhouettes show where disassembled components should be placed. safety. A virtual tablet, which is propped up on one side of the workbench, reflects these instructions in written form. Highlights and animated hints: When training at beginne level, the objects of immediate interest are highlighted with a yellow glow (Figure 3, B). This makes the objects pop out leveraging preattentive visual processing [3]. Furthermore, the required action is repeatedly demonstrated by an animated virtual hand (e.g., twisting a component to loosen it, as shown in Figure 3, B).

Haptic feedback for tool use: Important haptic cues, which are typically implicitly provided by the physical objects and tools used, were simulated through vibration of the Vive controllers. An example of this is the clicking of a torque screw driver, when the screw was fastened beyond its intended torque. This was accompanied by a recorded clicking sound of the torque screwdriver.

Indication of progress: When manipulation of an object required repeated or prolonged interaction (e.g., fastening a screw, or holding down a button for 2 seconds), a radial progress bar provided the user with feedback.

Snapping: To better facilitate interaction with the small virtual object components, we implemented snapping on two occasions: 1. When disassembling the dosing pump the user must place components in predefined locations on the workbench (see Figure 3, C). Components snap to these locations when released in proximity. 2 . When reassembling the dosing pump, components will snap to their correct position and orientation, when held and released very close to where they should be mounted.

\section{Evaluation}

A user study was carried out to compare VR Training to traditional training methods for performing maintenance on the dosing pump, and to test the following hypotheses: (H1) VR Training enables participants to successfully per- form a structured maintenance task in the real world; $(\mathrm{H} 2)$ VR Training and Pairwise Training are comparable with regards to (a) Task Completion Time and (b) Number of Errors; $(\mathrm{H} 3)$ VR Training and Video Training are comparable with regards to (a) Task Completion Time and (b) Number of Errors; (H4) During the task, participants have higher (c) Confidence in Actions after VR Training compared to Video Training. We further endeavored to explore which parameters could be used to evaluate the effectiveness of $V R$ Training in comparison to traditional training methods, and what design decisions critically impact the learning outcome in a $V R$ Training simulation for a maintenance task.

\section{Participants and Study Design}

A total of 36 volunteers (4 female) participated in the study. These were selected from among the experimenters' social network and the campus of Aarhus University. None of the participants had prior experience with dis/assembling dosing pumps, but $8 / 36$ claimed at least some experience with mechanical and electronic maintenance. In the VR Training group 11/12 participants had experienced VR on prior occasions. Participants were quasi-randomly divided into three groups (12 participants per group, gender-balanced): (1) Pairwise Training group, (2) Video Training group, or (3) VR Training group (shown in Figure 1, A, B and $\mathrm{C}$ respectively). In all training conditions, participants were instructed to perform the maintenance task by following the steps presented in Table 1. The Pairwise Training group was guided through the steps by a researcher (always the same) who acted as instructor. The Video Training group learned the task by watching the instructional video. Both the Pairwise Training group and the Video Training group performed each of the steps on an actual dosing pump. The VR Training group was instructed in VR and completed the task on a virtual dosing pump. 


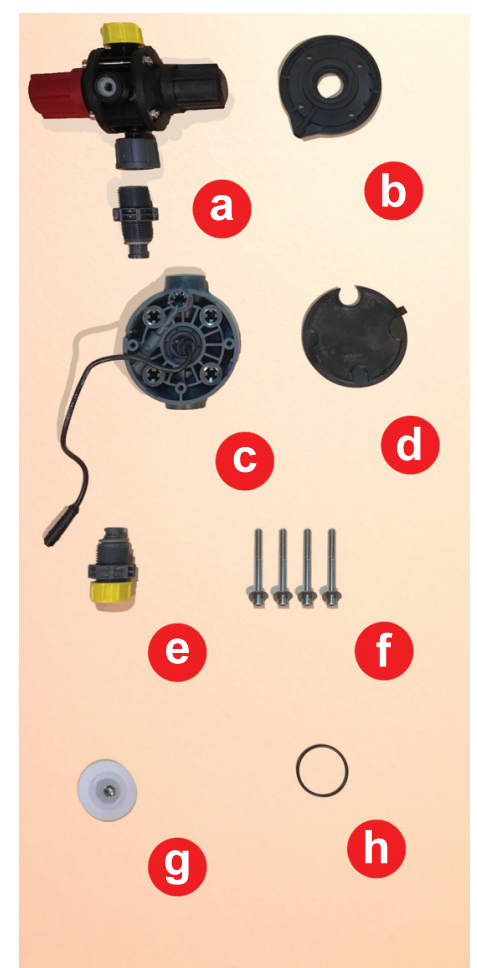

Figure 4: Components of the disassembled dosing pump: (a) Top ball valve (including the multi-function valve); (b) Drainer; (c) Dosing head; (d) Back lid; (e) Bottom ball valve; (f) Screws; (g) Diaphragm; (h) o-ring.
After training, participants performed the task without guidance in a post-test. Here we recorded (a) Task Completion Time, (b) Number of Errors, and (c) Confidence in Actions. Task Completion Time was measured from when participants first started performing maintenance, to when they completed the task - excluding time for donning safety gear, since participants spent a varying amount of time on this, and it was not deemed critical to the task

Number of Errors was counted based on situations when participants did not perform a step correctly and did not correct the error right away. These were registered on a checklist of possible errors, which were previously identified by a domain expert in a pilot study.

Confidence in Actions was given as subjective rating on a 5-point Likert scale.

\section{Apparatus}

Both the Pairwise Training and Video Training required a dosing pump and an extra o-ring that needed to be replaced (see components of a disassembled dosing pump in Figure 4). Furthermore, safety glasses, gloves, a torque screwdriver and a needle tool were provided. The needle tool was used to remove the o-ring in the dosing pump. For the VR Training, participants were seated at a desk and provided with an HMD and two controllers. The VE included a virtual dosing pump, torque screwdriver, needle tool and safety gear, in correspondence with the materials required for the real-world task.

The post-test was conducted in a different room on a dosing pump of the same model. However, the setup differed in that the dosing pump was connected to a larger system including two other pumps and several pipes. The necessary tools and safety gear were again provided.
Procedure

After giving their informed consent and agreeing to video recording and anonymous data collection, participants provided demographic information and details about prior experience with VR, technical skills etc. Then each participant could inspect the tools and setup that would be used in the post-test. Participants were shown the dosing pump, and the components that needed to be dis/assembled in the task were pointed out (see Figure 4).

Independent of the condition, the training always involved the same 19 steps for the maintenance task (Table 1) and took approximately 20 minutes. During this time participants trained the task twice. In the Pairwise Training group the experimenter provided instructions from a manuscript. These included explanations for why certain actions were necessary and how different parts should be oriented. On the first run-through, the participant typically relied heavily on the instructor's guidance. On the second run-through they remembered many required actions and the instructor provided less guidance. However, critical actions, such as using safety equipment, were always mentioned. In the Video Training group participants were guided through the maintenance task by a video shown on a PC, while performing the steps on the physical dosing pump. They were allowed to pause the video and replay parts if necessary, but no other aids were given. In the VR Training group participants first spent about 5 minutes in a VR "playground" [2], to get familiar with the VE. They could also ask questions and request assistance during this introduction phase. Then followed the actual training simulation, first with guidance level set to Beginner, and then to Intermediate.

When the training was completed, each participant spent 6 minutes on a mental rotation test [9]. This test intended to distract the participant and diminish the recency knowledge effect between the training session and the post-test [2]. In 

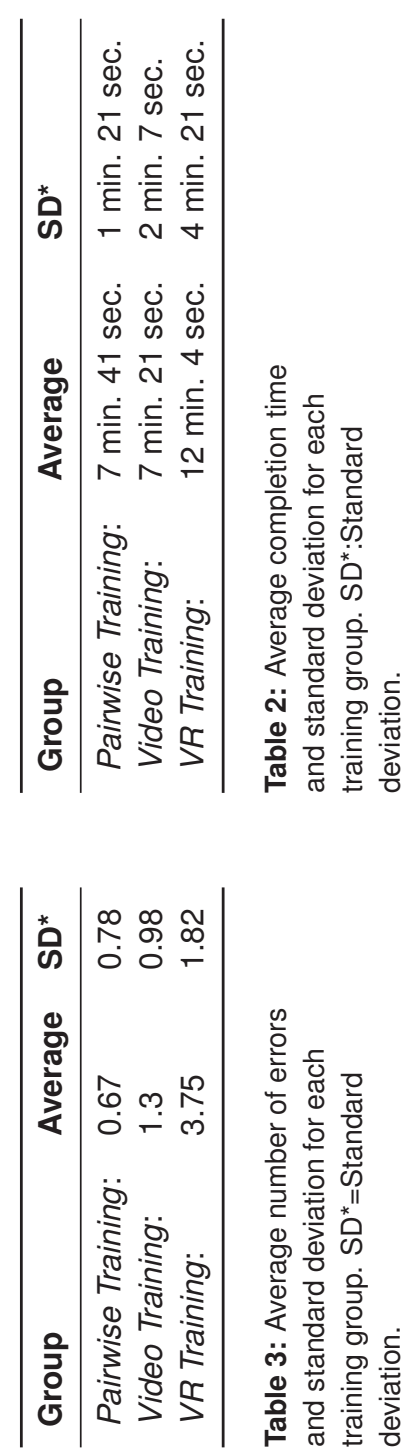

the post-test the participants were asked to replace the oring on a physical dosing pump without assistance, to evaluate how well the respective training type prepared them for the task. Participants were informed that Task Completion Time and Number of Errors would be measured. An experimenter was present to observe and assist in case of severe breakdowns. In a concluding semi-structured interview, participants were asked how confident they felt while performing the task in the post-test. The overall study duration for each participant was approximately one hour.

\section{Findings}

Objective and subjective task performance

Three performance measures were recorded during the post-test: (1) Task Completion Time and (2) Number of Errors provided objective measures, whereas (3) Confidence in Actions was subjective. To test for significant differences between the training types, a Kruskal-Wallis test was carried out $(\alpha=0.05)$. This non-parametric test was also used for Task Completion Time, since Bartlett's test showed a violation of homogeneity of variances $\left(\chi^{2}=12.852, p<\right.$ 0.01 ). Pairwise comparisons were made by post-hoc MannWhitney test with Bonferroni correction.

Task Completion Time and Number of Errors: The average Task Completion Times are visualized in Figure 5 (A), and given in Table 2. On average participants took about 1.6 times longer for the maintenance task in the post-test after VR Training, compared to both other groups. This effect is significant $\left(\chi^{2}=12.65, p<0.01\right.$; Posthoc: VR Train ing - Pairwise Training $p<0.01, r=0.61 \mid$ VR Training Video Training $p<0.01, r=0.62$ ).

There is also a significant effect of training type on Number of Errors $\left(\chi^{2}=17.44, p<0.01\right)$, where pairwise comparisons again showed differences between the VR Training and both the Pairwise Training ( $p<0.01, r=0.76)$ and the Video Training $(p<0.01, r=0.63)$. As is evident from Figure $5(\mathrm{~B})$ and the values in Table 3, the VR Training group made more than 3 errors on average, while both other groups made about 1 .

A Pearson's correlation test shows a positive association between Task Completion Time and Number of Errors $(r=$ $0.73, p<0.01)$. We found no connection between the task performance and participants' prior technical expertise.

Confidence in Actions: As illustrated in Figure 5 (C), the participants' Confidence in Actions may be a reliable indicator of their objective task performance, since Pairwise Training and Video Training generally led to higher confidence than $V R$ Training. This effect was again significant $\left(\chi^{2}=9.38, p<0.01\right.$; Posthoc: VR Training - Pairwise Training $p<0.01, r=0.57 \mid V R$ Training - Video Training $p<0.01, r=0.49$ ).

Common types of Errors

Based on direct observation and analysis of video recordings, the following errors occurred most commonly.

Orientation error: When reassembling the dosing pump some participants had difficulty figuring out the correct orientation of components, such as the drainer and dosing head (Figure 4, b and c). In the VR Training group 5/12 participants required multiple attempts to make components fit, versus 2/12 in the Video Training group and 0/12 in the Pairwise Training group.

Sequential error: Another common error was that participants forgot a step and proceeded to the next one, e.g., forgetting to remove the top and bottom ball valves (Figure 4, a and e). This happened most frequently in the VR Training group (7/12), compared to $1 / 12$ in the Pairwise Training group and 0/12 in the Video Training group.

Safety issue: The third, and perhaps most critical error, was neglecting the safety equipment, e.g., not putting on 


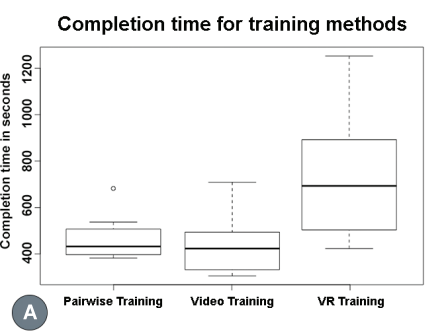

Number of errors for training methods

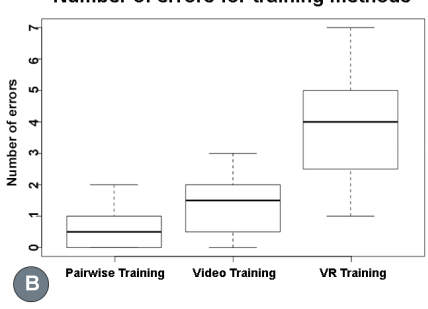

Level of confidence for training methods

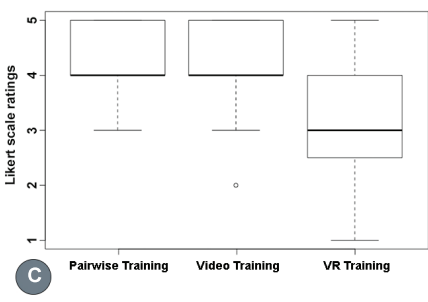

Figure 5: Boxplots of the three evaluation parameters: (A) Task Completion Time (B) Number of Errors, and (C) Confidence in Actions (5-point Likert scale) for each training method. the safety glasses. Here both the Video Training group (4/12) and the VR Training group (3/12) were more negligent than the Pairwise Training group (1/12).

\section{Discussion}

We expected participants trained in VR to be capable of performing a maintenance task on a real-world dosing pump, since they learned the same sequence of steps in a high-fidelity simulation (H1). This hypothesis was supported: all participants in the VR Training group were able to perform the task after just 20 minutes of training. VR training has the benefits of being scalable to service multiple trainees at the same time and train many tasks on an arbitrary number of machines in various environments and configurations, while not requiring the availability of specific equipment, apart from the VR setup. However, the success of this medium is arguably task-dependent, and it remains to be explored what kind of skill acquisition is best supported by VR training.

Based on the opinion of training experts, we expected the three training methods to yield comparable task performance ( $\mathrm{H} 2$ and $\mathrm{H} 3)$. Our results do not support these hypotheses, since in the post-test the overall performance (Task Completion Time, Number of Errors) was significantly better in both Pairwise Training and Video Training compared to VR Training. This does not reflect the promising results of earlier work [7] and may suggest that the richness of hands-on experience, which is very difficult to simulate in VR, presents a great benefit to learning. The hands-on component may also be key to the success of AR-based training simulations [10]. A further contributing factor may be contextual changing, which is arguably lower in handson training methods, since training environment and equipment do not vary between training and test scenarios [6].
Lastly, we expected that VR Training would instill a higher level of confidence than Video Training, since multimodal instructions presented in an interactive 3D-environment are richer and provide a better understanding of what to do, compared to an instructional video seen on a 2D display (H4). This hypothesis was flawed, since it is based solely on how instructions are presented, and does not consider how the trainee follows these instructions: While in VR Training participants manipulated a virtual pump, in Video Training participants could explore the physical dosing pump and learn through trial and error. While the comparison of VR Training with two training types that involve hands-on experience may be unfair, these present the training methods that are currently applied for such tasks and $V R$ Training is widely being considered equally effective. Our evaluation may serve to highlight limitations of $V R$ Training and demonstrate how hands-on experience impacts the learning process.

Simulating hands-on experience in VR: The presented VR training takes a first step towards simulating hands-on experience in VR by requiring tool use and realistic hand motions (e.g., turning wrist to tighten a screw), as well as providing haptic feedback through vibrations of the controller and clear visual indications of progress. However, our results show that still richer multimodal feedback needs to be provided, in particular for critical cues such as components snapping together only when assembled in correct orientation. Future research should explore delivering richer haptics e.g., through force feedback [4].

Balancing the level of challenge When designing a $V R$ Training tool, it is important to ensure effective step-by-step guidance, while setting an adequate level of challenge to foster learning. Due to the extensive amount of hints given in the first VR training session (beginner level), users might not have reached the cognitive stage of skill acquisition. 
Therefore, we reduced the level of guidance in the second session (intermediate level). Future work could explore more or dynamic levels of difficulty. Further, the snapping of components into correct orientation may have hindered learning. Future research could focus on supporting learning through trial and error, as well as repeated/prolonged VR training sessions [2] with test phases in between.

\section{Conclusion}

This case study presents a VR Training simulation for a maintenance task on a dosing pump and an evaluation thereof in comparison to traditional training methods applied at Grundfos (Pairwise Training, Video Training). The three experimental groups were compared based on the participants' performance in terms of (a) Task Completion Time, (b) Number of Errors, and (c) Confidence in Actions. Study results reveal that $V R$ Training was effective in teaching the training task. However, both hands-on training methods, Pairwise Training and Video Training, led to significantly better performance than VR Training.

\section{Acknowledgements}

We would like to thank Grundfos for generously supplying the material needed to explore this case, and express our gratitude to all study participants. This work was partially supported by the Innovation Fund Denmark (IFD grant no. 6151-00006B), within the MADE Digital project of the Manufacturing Academy of Denmark.

\section{REFERENCES}

[1] Andrew C Boud, David J Haniff, Chris Baber, and SJ Steiner. 1999. Virtual reality and augmented reality as a training tool for assembly tasks. (1999), 32-36.

[2] Patrick Carlson, Anicia Peters, Stephen B Gilbert, Judy M Vance, and Andy Luse. 2015. Virtual training:
Learning transfer of assembly tasks. IEEE transactions on visualization and computer graphics 21, 6 (2015).

[3] Christopher Healey and James Enns. 2011. Attention and visual memory in visualization and computer graphics. IEEE transactions on visualization and computer graphics 18, 7 (2011), 1170-1188.

[4] Ronan Hinchet, Velko Vechev, Herbert Shea, and Otmar Hilliges. 2018. DextrES: Wearable Haptic Feedback for Grasping in VR via a Thin Form-Factor Electrostatic Brake. In The 31st Annual ACM Symposium on User Interface Software and Technology. ACM, 901-912.

[5] Jason Jerald. 2015. The VR book: Human-centered design for virtual reality. Morgan \& Claypool.

[6] JJ Kozak, PA Hancock, EJ Arthur, and ST Chrysler. 1993. Transfer of training from virtual reality. Ergonomics 36, 7 (1993), 777-784.

[7] A Langley, G Lawson, S Hermawati, M D'Cruz, J Apold, F Arlt, and K Mura. 2016. Establishing the usability of a virtual training system for assembly operations within the automotive industry. Human Factors \& Ergonomics in Manufacturing \& Service Industries 26, 6 (2016).

[8] Robert J Seidel and Paul R Chatelier. 2013. Virtual reality, training's future?: perspectives on virtual reality and related emerging technologies. Vol. 6. Springer Science \& Business Media.

[9] Steven G Vandenberg and Allan R Kuse. 1978. Mental rotations, a group test of three-dimensional spatial visualization. Perceptual and motor skills 47, 2 (1978).

[10] Stefan Werrlich, Austino Daniel, Alexandra Ginger, Phuc-Anh Nguyen, and Gunther Notni. 2018. Comparing HMD-Based and Paper-Based Training. 